\title{
Previsões de Vazões Mensais na Bacia Hidrográfica do Xingu - Leste da Amazônia
}

\author{
Edmundo Wallace Monteiro Lucas ${ }^{1,2}$ (D), Francisco de Assis Salviano de Sousa ${ }^{1}$ (D), \\ Fabrício Daniel dos Santos Silva ${ }^{3}$ (D), Rodrigo Lins da Rocha Júnior ${ }^{3}$ (D), \\ Kleber Renato da Paixão Ataide ${ }^{2}$ \\ ${ }^{1}$ Unidade Acadêmica de Ciências Atmosféricas, Universidade Federal de Campina Grande, \\ Campina Grande, PB, Brasil. \\ ${ }^{2}$ Instituto Nacional de Meteorologia, Brasília, DF, Brasil. \\ ${ }^{3}$ Instituto de Ciências Atmosféricas, Universidade Federal de Alagoas, Maceió, AL, Brasil.
}

Recebido em: 29 de Junho de 2020 - Aceito em: 1 de Setembro de 2020

\begin{abstract}
Resumo
Modelos empíricos chuva-vazão vêm sendo cada vez mais usados nas últimas décadas, devido a indisponibilidade de dados de entrada dos modelos conceituais, e à confiabilidade, rapidez, e menor complexidade destes modelos. Neste contexto, foi aplicada a técnica de Regressão por Componentes Principais (RCP) na simulação das vazões médias mensais de oito estações fluviométricas na Bacia Hidrográfica do Xingu (BHX), pertencente a grande bacia hidrográfica amazônica. Na BHX se encontra a usina hidroelétrica de Belo Monte, além de importantes áreas de preservação ambiental. Demonstra-se na pesquisa o grau de relação entre a precipitação e vazão, entrada e resposta do modelo chuva-vazão baseado em RCP, e os respectivos graus de defasagem com eficiência preditiva. A RCP apresentou bons resultados na simulação das vazões mensais em todas as estações selecionadas, caracterizando bem a dinâmica das séries temporais com resultados ótimos nos períodos de estiagem (maio a outubro) e tendência a leve subestimativa nos períodos chuvosos (novembro a abril). Tais resultados utilizando a precipitação observada na simulação das vazões da BHX permitem concluir que um bom sistema de previsão climática para a precipitação sazonal poderá inferir um grau preditivo importante para as vazões com até três meses de antecedência.
\end{abstract}

Palavras-chave: dinâmica do clima, modelo chuva-vazão, regressão por componentes principais.

\section{Monthly Streamflow Forecast in the Xingu River Basin - Eastern Amazon}

\begin{abstract}
Empirical rainfall-streamflow models have been increasingly used in recent decades, due to the unavailability of input data from conceptual models, and the reliability, speed, and less complexity of these models. In this context, the Principal Component Regression technique (PCR) to simulate the monthly average streamflow of eight river stations in the Xingu River Basin (XRB) was applied, which belongs to the large Amazon basin. The XRB has the Belo Monte hydroelectric plant, as well as important areas of environmental preservation. The degree of relationship between rainfall and streamflow, input and response of the rainfall-streamflow model based on PCR is shows in the research and the respective degrees of lag with predictive efficiency. The PCR showed good results in the simulation of the monthly streamflow in all the selected stations, characterizing well the dynamics of the time series with excellent results in the dry periods (May to October) and a tendency to slightly underestimate in the rainy periods (November to April). These results, using the rainfall observed for streamflow simulation in the XRB, allow us to conclude that a good climate forecasting system for seasonal rainfall can infer an important predictive degree for streamflow up to three months in advance.
\end{abstract}

Keywords: climate dynamics, rainfall-streamflow model, principal component regression.

Autor de correspondência: Edmundo Wallace Monteiro Lucas, edmundowallace@gmail.com. 


\section{Introdução}

Os modelos hidrológicos chuva-vazão (conceituais ou empíricos) procuram representar a parte terrestre do ciclo hidrológico, transformando a precipitação que cai sobre a bacia em vazão no rio (Almeida e Serra, 2017). Estes modelos são amplamente utilizados para extrapolar séries temporais de vazões no tempo e no espaço para aplicações operacionais e científicas, tais modelos permitem estender os registros de vazões disponíveis sendo possível prever o comportamento hidrológico das bacias hidrográficas para diferentes aplicações e cenários climáticos (Wagner e Whater, 2006).

Em geral os modelos hidrológicos aplicados levam em conta a relação entre as variáveis, sendo classificados em empíricos ou conceituais. Os modelos empíricos capturam relações entre a variável preditora e a variável observada, sem necessariamente representar o comportamento físico dos processos envolvidos, que são geralmente representados pelos modelos conceituais (Tucci, 2005; Todini, 2007; Devi et al., 2015).

Há um universo de possibilidades na utilização de modelos empíricos como: regressões múltiplas, redes neurais, modelos auto-regressivos com médias móveis (ARMA e ARIMA), análises de componentes principais (Malfatti et al., 2018). Estes modelos levam alguma vantagem na aplicação em relação aos modelos conceituais, pois em geral, utilizam um número menor de dados de entrada na modelagem sendo de fácil ajuste e validação. Muitos estudos foram aplicados com estes modelos nas simulações de vazões em bacias hidrográficas (Hu et al., 2007; Remesan et al., 2009; Alexandre, 2012; Malfatti et al., 2014; Filho e Lall, 2014; Remesan et al., 2018; Malfatti et al., 2018).

$\mathrm{Na}$ técnica de regressão por Analises de Componentes Principais, em vez de regredir diretamente a variável dependente na variável explicativa, as componentes principais da variável explicativa são usados como regressores, ortogonalizando o problema da regressão e tornando os cálculos mais fáceis e estáveis (Jolliffe, 1982). Alguns trabalhos vêm utilizando modelos hidrológicos com a técnica de Análise de Componentes Principais nas simulações de vazões (Hu et al., 2007; Duan et al., 2010; Remesan et al., 2018).

Duan et al. (2010), desenvolveram um modelo de regressão regional para a bacia do Rio Hailar na China correlacionado o escoamento anual com dados de precipitação, evaporação e outras características da bacia para o período de 1956 a 2006. Essa bacia é a principal fonte de água para indústria e agricultura local. Na elaboração dos resultados foi utilizado a técnica de regressão por analises de componentes principais, os resultados mostraram que o modelo se mostrou robusto, pois os escoamentos previstos são compatíveis com os correspondentes observados apresentando uma correlação satisfatória. Esta técnica, pode ajudar a resolver problemas de multicolinearidade na correlação entre o escoamento e as características da bacia durante as simulações hidrológicas.

A aplicação de modelagem hidrológica chuva-vazão por meio de técnicas estatísticas (modelos empíricos) vem crescendo nas últimas décadas, principalmente pela indisponibilidade de alguns dados de entrada dos modelos conceituais, a necessidade de previsões menos complexas, mais rápidas e confiáveis. As bacias hidrográficas localizadas na Amazônia são particularmente adequadas para a aplicação de modelos hidrológicos chuva-vazão pois a precipitação e a vazão são altamente correlacionadas nestas bacias.

A precipitação é a variável meteorológica que mais influencia o fluxo dos rios, portanto, mudanças nos padrões da precipitação podem impactar as atividades socioeconômicas da região como agricultura, navegação e a geração de energia elétrica. Porém, esses impactos também variam de acordo com outras características da bacia como o tipo de vegetação e o uso e cobertura do solo (Coe et al., 2009; Dias et al., 2018).

A Bacia Hidrográfica do Xingu (BHX) está localizada no Amazônia Brasileira e abriga hoje um dos maiores projetos do governo brasileiro em termos de produção de energia, o Complexo Hidrelétrico de Belo Monte o maior empreendimento energético $100 \%$ brasileiro. A hidroelétrica de Belo Monte tem capacidade potencial instalada de produzir $11.233 \mathrm{MW}$, isto representa quase $10 \%$ da demanda brasileira de energia elétrica (Stickler et al., 2013; De Menezes, 2017; Norte Energia, 2018). Além do potencial para geração de energia hidrelétrica, a BHX também tem extrema importância ambiental e cultural, por conter além da floresta amazônica, porções consideráveis do cerrado, unidades de conservação florestal e de povos indígenas (Villas-Boas, 2012).

O objetivo deste trabalho é aplicar a modelagem hidrológica chuva-vazão na simulação da vazão mensal nas estações fluviométricas da bacia Hidrográfica do Xingu no Leste da Amazônia Brasileira, utilizando a técnica de Regressão por Componentes Principais. Os resultados desta pesquisa buscam contribuir significativamente com previsões hidrológicas mensais mais rápidas e confiáveis e apresentar a metodologia como uma ferramenta útil e operacional, para auxiliar e apoiar a tomada de decisões em setores-chave, como gerenciamento de grandes projetos hidraulicos (geração de energia hidrelétrica) e no planejamento dos usos múltiplos dos recursos hídricos na BHX.

\section{Materiais e Métodos}

\section{1. Área de estudo}

A BHX (Fig. 1) importante sub-bacia da Bacia Amazônica localizada nos Estados do Pará e Mato Grosso, possui uma área de drenagem de aproximadamente 

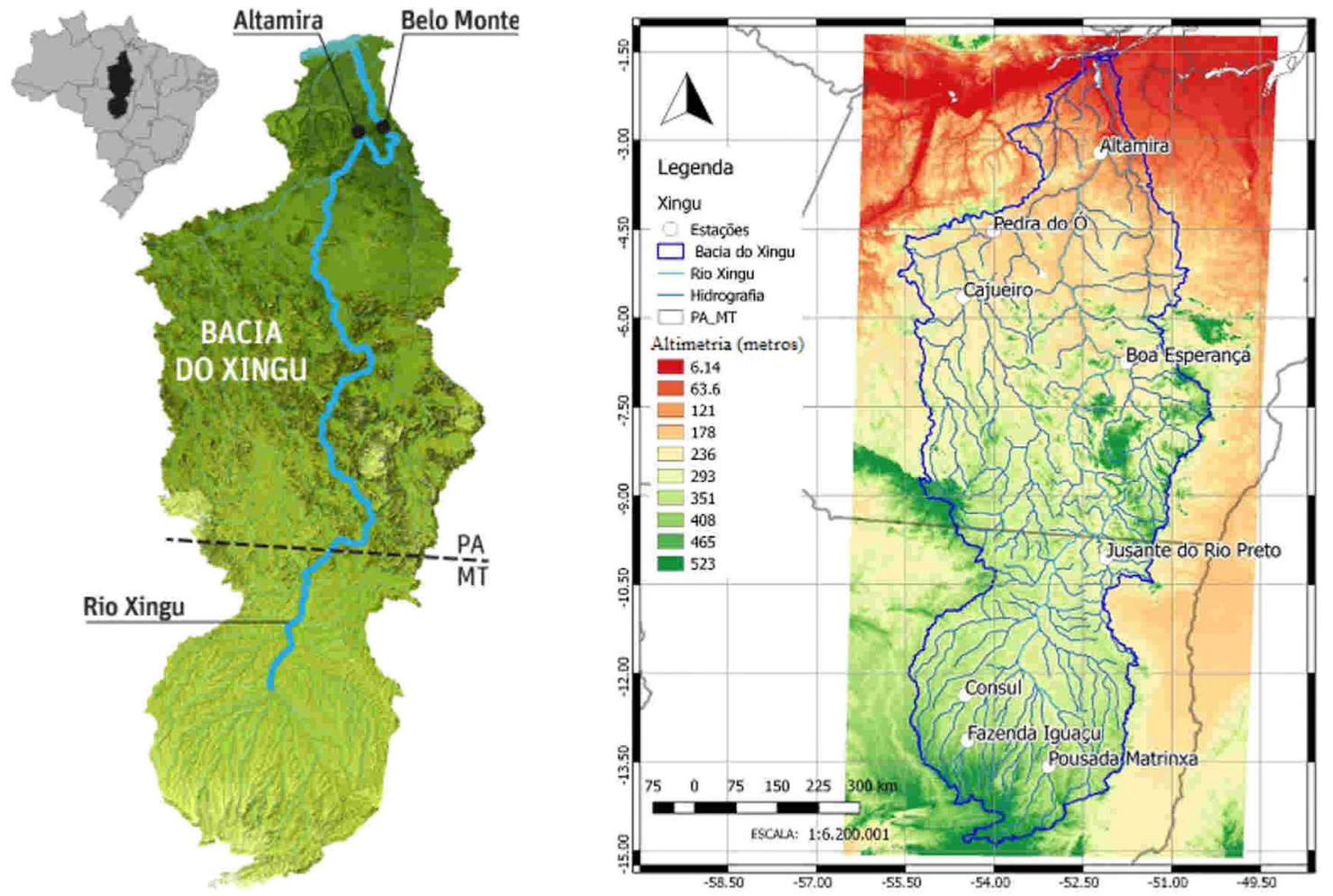

Figura 1 - Localização geográfica da Bacia do Xingu e das estações fluviométricas da ANA.

$510.000 \mathrm{~km}^{2}$. O rio principal Xingu, nasce em Mato Grosso no encontro das Serras Formosa e Roncador no cerrado brasileiro e se uni ao bioma amazônico percorrendo uma extensão de aproximadamente $2.600 \mathrm{~km}$ (Silva et al., 2013). A BHX está dividida basicamente em três regiões: Alto Xingu, Médio Xingu e Baixo Xingu. Os principais afluentes do rio Xingu são os rios Culuene, Fresco, Curuá, Iriri e Bacajá (CPRM, 2019).

A variabilidade temporal e espacial da precipitação na BHX apresenta um regime um pouco diferenciado entre as partes norte e sul da bacia. De um modo geral os maiores volumes de precipitação ocorrem entre novembro a abril e o período de estiagem entre maio e outubro. $\mathrm{Na}$ parte sul e centro da bacia os maiores volumes de precipitação são observados nos meses de dezembro, janeiro e fevereiro, oriundos da Zona de Convergência do Atlântico Sul (ZCAS). Já na parte norte os maiores volumes de precipitação são observados nos meses de janeiro, fevereiro e março, nesse período a parte norte da BHX está sobre influência da Zona de Convergência Intertropical ZCIT, principal sistema meteorológico precipitante na Amazônia (Reboita et al., 2010; Santos et al., 2015; Santos et al., 2016).

\subsection{Dados utilizados}

Foram selecionadas para este trabalho estações fluviométricas com dados consistidos de vazões médias mensais no período de 1980 a 2016 localizadas na BHX (Fig. 1). As estações foram selecionadas a partir do Inventário de Estações Fluviométricas da Agência Nacional de Águas (ANA, 2019) e extraídos dados com o software HidroWeb (versão 3.1.1). Informações como município, coordenadas geográficas, rio, área drenada e período de dados das estações selecionadas estão descritas na Tabela 1. A estação fluviométrica de Altamira no rio principal Xingu, no exutório da BHX (montante do Reservatório de Belo Monte), será usada como referência na validação dos resultados das simulações das vazões mensais.

Foram utilizados dados diários de precipitação das análises gradeadas disponibilizados por Xavier et al. (2016), no período de 1980 a 2016. Estes dados fazem parte de um projeto conjunto entre a Universidade do Texas (USA) e Universidade Federal do Espírito Santo (Brasil), estão disponíveis em uma grade com resolução espacial de $0,25^{\circ} \times 0,25^{\circ}$ para todo o território brasileiro. Com intuito de comparar e apresentar os resultados do trabalho, foram utilizados ainda totais mensais de precipitação observados na estação meteorológica do INMET em Altamira.

\subsection{Regressão por Componentes Principais}

A Regressão por Componentes Principais (RCP), foi inicialmente proposta por Kendall (1975) e Hotelling (1975). Essa técnica de regressão está baseada em Aná- 
Tabela 1 - Informações adicionais referentes as estações fluviometricas na BHX.

\begin{tabular}{|c|c|c|c|c|c|c|c|}
\hline Estação & Município & UF & Lat $\left({ }^{\circ}\right)$ & Long $\left({ }^{\circ}\right)$ & Rio & Área $\left(\mathrm{km}^{2}\right)$ & Período \\
\hline Altamira $^{*}$ & Altamira & $\mathrm{PA}$ & $-3,21$ & $-52,21$ & Xingu & 448.000 & $1980-2014$ \\
\hline Pedra do Ó & Altamira & PA & $-4,53$ & $-54,01$ & Iriri & 122.000 & $1980-2014$ \\
\hline Cajueiro & Altamira & PA & $-5,65$ & $-54,52$ & Curuá & 35.600 & $1980-2016$ \\
\hline Boa Esperança & São Felix do Xingu & PA & $-6,75$ & $-51,77$ & Fresco & 42.400 & $1980-2016$ \\
\hline Jusante do Rio Preto & Vila Rica & MT & $-10,05$ & $-52,11$ & Comandante Fontoura & 12.000 & $1980-2014$ \\
\hline Consul & Feliz Natal & MT & $-12,37$ & $-54,49$ & Van den Steinen & 8.900 & $1980-2014$ \\
\hline Fazenda Itaguaçu & Paranatinga & MT & $-13,14$ & $-54,45$ & Rio Ronuro & 3.840 & $1980-2016$ \\
\hline Pousada Matrinxa & Canarana & MT & $-13,57$ & $-53,08$ & Culuene & 11.600 & $1980-2016$ \\
\hline
\end{tabular}

*Estação de referência no exutório da BHX, utilizada na validação do modelo chuva-vazão.

lises de Componentes Principais (ACP), no intuito de contornar dificuldades encontradas na Regressão Linear Múltipla (RLM), ou seja, eliminando problemas de multicolinearidade das variáveis, e também reduzindo a quantidade de ruído inserido no modelo. A RCP é frequentemente aplicada como método de redução de dimensionalidade sem acarretar perdas significativas de informações que estão contidas nos dados (Otto, 1999).

$\mathrm{Na}$ técnica de RCP em vez de regredir diretamente a variável dependente na variável explicativa, as componentes principais da variável explicativa são usados como regressores. Frequentemente, as componentes principais com altas variâncias (aquelas baseadas em autovetores correspondentes aos autovalores da matriz de variânciacovariância da amostra das variáveis explicativas) são selecionados como regressores. No entanto, as componentes principais com baixas variações também podem ser importantes na regressão.

O detalhamento matemático sobre a aplicação desta técnica de análise estatística multivariada está descrita em Bishop (2006) e Izenman (2008). As premissas da técnica de regressão por componentes principais são as mesmas usadas na regressão linear múltipla, como: linearidade, variação constante e independência. Como a regressão por componentes principais não fornece limites de confiança, a normalidade não precisa ser assumida.

Os modelos aplicados de RCP podem ser escritos seguindo uma notação usual (Eq. (1)), suponha que a equação de regressão possa ser escrita em forma de matriz como:

$$
Y=X B+r
$$

onde $(Y)$ é a variável dependente, $(X)$ representa a variável independente, $(B)$ é o coeficiente de regressão a ser estimado e $(r)$ representa o erro ou resíduo.

\subsection{Ferramenta de previsibilidade}

Nesta pesquisa foi utilizado o software Climate Predictability Tool (CPT), versão 15.6.3 na Regressão por Componentes Principais para simulações de vazões mé- dias mensais na BHX. Diversos trabalhos foram desenvolvidos aplicando esta ferramenta de previsão climática, muito utilizada nos Serviços Meteorológico Nacionais e Instituições de Pesquisa (Lucio et al., 2010; Kipkogei et al., 2017; Esquivel et al., 2018; Landman et al., 2019).

A Ferramenta CPT é um software desenvolvido pelo International Research Institute for Climate and Society IRI (Columbia University), voltado para construção de modelos de previsão climática sazonal, simulações e validações de modelos com diferentes dados atualizados. Embora a ferramenta seja especificamente projetada para essas aplicações, ela pode ser usada em configurações mais gerais com qualquer dado de entrada utilizando basicamente três métodos de simulação: Análise de Correlação Canônica, Regressão de Componentes Principais ou Regressão Linear Múltipla para qualquer aplicação (IRI, 2019; Mason e Tippett, 2017).

O CPT utiliza dois conjuntos de dados nas simulações. O primeiro conjunto de dados contém as "variáveis X", chamadas de "preditores", "variáveis independentes" ou "variáveis explicativas". Essas variáveis X são usadas para prever as variáveis no segundo conjunto de dados, que deve conter as "variáveis Y", que são chamadas de "preditandos", "variáveis dependentes" ou "variáveis de resposta". Neste trabalho foram utilizados os dados mensais de chuva como variáveis explicativa e as vazões mensais como variáveis de resposta.

A variável preditora (precipitação mensal) usada para prever a vazão mensal, foi obtida dos dados em grade selecionados na BHX, no período de 1980 a 2016 (Xavier et al., 2016). Essa base de dados em grade com resolução espacial de $0,25^{\circ}$ x $0,25^{\circ}$ (Xavier et al., 2016), vem sendo amplamente utilizada para o Brasil como fonte de informações meteorológicas para pesquisas e aplicações diversas (Da Silva et al, 2018; Da Silva et al, 2019; ParedesTrejo et al., 2019). Para tanto, foi necessário definir o número mínimo e máximo de modos da RCP, sendo estes limitados aos mesmos valores de números mínimos e máximos das FOE (Funções Ortogonais Empíricas) das variáveis preditora (precipitação) e resposta (vazão). $\mathrm{Na}$ prática, o CPT tem apresentado melhores resultados com a 
seleção máxima de 10 modos para RCP e FOE (Lucio et al., 2010), essa orientação foi seguida neste trabalho. Durante a simulação a ferramenta determina o número ótimo dos modos acima para cada simulação mensal.

\subsection{Avaliadores de desempenho}

Para avaliar o desempenho da aplicação da técnica de RCP nas simulações das vazões mensais na BHX, foram utilizadas algumas funções objetivas no processo de treinamento/calibração e validação. Foram calculados parâmetros estatísticos para avaliação de desempenho do modelo como: Coeficiente de Correlação ou Correlação de Pearson $(C C)$. Apresentado conforme equação abaixo (Eq. (2)):

$$
C C=\frac{\sum_{i=1}^{n} x_{i} y_{i}-\frac{1}{n}\left(\sum_{i=1}^{n} x_{i}\right)\left(\sum_{i=1}^{n} y_{i}\right)}{\sqrt{\sum_{i=1}^{n} x_{i}^{2}-\frac{1}{n}\left(\sum_{i=1}^{n} x_{i}\right)^{2}} \sqrt{\sum_{i=1}^{n} y_{i}^{2}-\frac{1}{n}\left(\sum_{i=1}^{n} y_{i}\right)^{2}}}
$$

onde $(x)$ e $(y)$ são as variáveis padronizadas a serem correlacionadas. O valor do $(C C)$ varia entre $-1 \leq \mathrm{r} \leq 1$. Quando $r$ tende ao valor 1, significa uma correlação perfeita positiva entre as duas variáveis, para r próximo a -1 há uma correlação negativa perfeita entre as duas variáveis, isto é, se uma aumenta, a outra sempre diminui e $r=0$ indica que as duas variáveis não dependem linearmente uma da outra.

A interpretação do coeficiente de correlação pode ser feita através de faixas, contudo, deve ser conhecer a natureza dos dados, tamanho da amostra e a relação entre as variáveis (Taylor, 1990). Em geral as faixas guias indicam correlação moderada para valores de $\mathrm{r}$ acima de 0,5 ; forte para valores acima de 0,7 e muito forte para valores acima de 0,9. Correlações fracas apresentam valores de $C C$ abaixo de 0,3 (Bozzoni et al., 2020).

Outras métricas estatísticas foram utilizadas na avaliação da simulação das vazões mensais incluindo o Coeficiente de Nash-Sutcliffe (CN) (Nash e Sutcliffe, 1970), $V I E S$ e a Raiz do Erro Quadrático Médio (REQM) (Wilks, 2006), definidos respectivamente nas Eqs. (3) a (5). Sendo $(F)$ os valores estimados pelo modelo, $(O)$ os valores observados a serem comparados, $(\bar{O})$ os valores médios observados e $(n)$ o número da amostra.

$$
\begin{gathered}
C N=1-\frac{\sum_{i=1}^{n}(O-F)^{2}}{\sum_{i=1}^{n}(O-\bar{O})^{2}} \\
V I E S=\frac{1}{n} \sum(F-O) \\
\text { REQM }=\sqrt{\frac{1}{n}} \sum(F-O)^{2}
\end{gathered}
$$

O coeficiente de Nash-Sutcliffe é frequentemente usado para avaliar o desempenho de modelos hidrológicos, podendo variar de $-\infty$ a 1 . O valor de $C N=1$ corresponde a um ajuste perfeito das vazões simuladas aos dados observados. Valores de $C N$ entre 0 e 1 geralmente são vistos como níveis aceitáveis de desempenho, enquanto valores $C N<0$ indicam que o valor médio observado é um preditor melhor do que o valor simulado, o que indica desempenho inaceitável. Valores entre $0,50<C N<0,65$ indicam um modelo de qualidade satisfatorio nas simulações de vazões (Moriasi et al., 2007).

Espera-se que o VIES seja sempre próximo de zero nos resultados, indicando uma boa simulação dos modelos aplicados. A Raiz do Erro Quadrático Médio (REQM), foi utilizado para tentar obter uma indicação quantitativa do erro do modelo em termos da quantidade dimensionada.

\subsection{Processo de calibração e validação}

Um passo importante realizado no trabalho, verificar se a técnica de RCP na modelagem chuva-vazão é capaz de representar de maneira precisa a vazão observada a partir das suas respectivas simulações. Para isso, o conjunto dos dados de chuva (variável preditora) e vazão (variável resposta) foram divididos em dois períodos distintos, primeiro período para treinamento/calibração do modelo chuva-vazão e o segundo período no processo de validação das simulações.

$\mathrm{Na}$ etapa de treinamento/calibração validações cruzadas entre as vazões mensais simuladas e observadas foram realizadas e calculado os coeficientes de correlação (CC), considerando cada mês e posto fluviométrico separadamente. Nesta etapa, as vazões médias mensais na BHX foram simuladas com a precipitação do mês atual e com a precipitação defasada de 1 a 3 meses, representadas por $t(0),(t-1),(t-2)$ e $(t-3)$, respectivamente. Esse procedimento foi realizado para obter entendimento intuitivo sobre o grau de relacionamento entre a precipitação e a vazão na BHX e para identificar o número apropriado de valores antecedentes de precipitação como entrada no modelo chuva-vazão para simulação da vazão mensal nas estações da bacia.

Identificado os valores apropriados antecedentes de precipitação mensal de cada estação fluviométrica na BHX, inicia-se o processo de validação das simulações, 
comparando os pares de previsão-observação do período não incluído no processo de calibração. A validação é feita com a avaliação das métricas estatísticas selecionados Coeficiente de Correlação $(C C)$, Coeficiente de Nash-Sutcliffe $(C N)$, VIES, Raiz do Erro Quadrático Médio $(R E Q M)$. Essa metodologia é usualmente utilizada na aplicação da modelagem chuva-vazão, visando obter o melhor arranjo empírico e desempenho do modelo (Câmara et al., 2016; Malfatti et al., 2018).

\section{Resultados e Discussão}

Os resultados nas análises das correlações cruzadas no período de treinamento/calibração entre 1981 a 2010, mostram que os melhores desempenhos nas simulações das vazões mensais na BHX foram obtidos com a precipitação mensal defasada no tempo $t(0)$ e $(t-1)$, os valores dos coeficientes de correlação encontrados nesta etapa são mostrados na Tabela 2. Se destacaram nas simulações das vazões mensais as estações fluviométricas de Pousada Matrinxa no tempo $t(0)$ e Altamira $(t-1)$, com os valores do coeficiente de correlação de 0,75 e 0,71 respectivamente. As simulações de vazões mensais com defasagens maiores na precipitação $(t-2)$ e $(t-3)$, apresentaram resultados menos satisfatórios em comparação as defasagens anteriores.

Conforme os resultados mostrados na Tabela 2, fica evidente que nas estações fluviométricas da BHX com grandes áreas de drenagem (Altamira, Pedra do Ó, Cajueiro, Boa Esperança e Jusante do Rio Preto) a vazão mensal em um determinado mês ( $\mathrm{t}$ ), está mais relacionada com as chuvas do mês anterior $(t-1)$ nas simulações realizadas. Estes resultados indicam que a contribuição da precipitação nestas localidades da BHX demora no máximo um mês para contribuir com a vazão mensal.

Já nas estações fluviométricas da BHX com área de drenagem menores, abaixo de $12.000 \mathrm{~km}^{2}$ (Consul, Fazenda Itaguaçu e Pousada Matrinxa), a vazão mensal em um determinado mês ( $t$ ) está mais relacionada com as chuvas do mesmo mês, ou seja, sem defasagem $t(0)$ nas
Tabela 2 - Coeficientes de Correlação $(C C)$ entre as vazões simuladas e observadas no período de treinamento, nas estações fluviométricas da BHX.

\begin{tabular}{|c|c|c|c|c|c|c|}
\hline Estação & Rio & $\begin{array}{l}\text { Área } \\
\left(\mathrm{km}^{2}\right)\end{array}$ & $t(0)$ & $\begin{array}{l}(t- \\
1) \\
\end{array}$ & $\begin{array}{l}(t- \\
2)\end{array}$ & $\begin{array}{l}(t- \\
3) \\
\end{array}$ \\
\hline Altamira ${ }^{*}$ & Xingu & 448.000 & 0,59 & 0,71 & 0,57 & 0,55 \\
\hline Pedra do Ó & Iriri & 122.000 & 0,60 & 0,70 & 0,56 & 0,53 \\
\hline Cajueiro & Curuá & 35.600 & 0,57 & 0,66 & 0,47 & 0,42 \\
\hline Boa Esperança & Fresco & 42.400 & 0,62 & 0,66 & 0,50 & 0,52 \\
\hline $\begin{array}{l}\text { Jusante do Rio } \\
\text { Preto }\end{array}$ & $\begin{array}{l}\text { Comandante } \\
\text { Fontoura }\end{array}$ & 12.000 & 0,54 & 0,64 & 0,53 & 0,50 \\
\hline Consul & Van den Steinen & 8.900 & 0,52 & 0,49 & 0,43 & 0,47 \\
\hline $\begin{array}{l}\text { Fazenda Ita- } \\
\text { guaçu }\end{array}$ & Rio Ronuro & 3.840 & 0,61 & 0,54 & 0,44 & 0,43 \\
\hline $\begin{array}{l}\text { Pousada } \\
\text { Matrinxa }\end{array}$ & Culuene & 11.600 & 0,75 & 0,50 & 0,49 & 0,42 \\
\hline
\end{tabular}

simulações do modelo, nestes casos, os resultados demostram que a contribuição da precipitação na vazão mensal ocorre dentro do mesmo mês. Estes resultados corroboram com metodologia similar adotada em outras bacias estudadas (Bravo et al., 2012 e Malfatti et al., 2018).

Definido acima o grau de relação entre a precipitação e a vazão mensal para cada estação fluviométrica da BHX, na Tabela 3 são mostrados os coeficientes de correlações para todos os meses separadamente no resultado do desempenho individual das estações fluviométricas na simulação das vazões mensais com a precipitação defasada no tempo $t(0)$ e $(t-1)$ no período de treinamento/ calibração. De uma maneira geral, a técnica de RCP aplicada conseguiu simular bem a vazão com base na precipitação em praticamente todos os meses do ano nas estações fluviométricas analisadas, contudo, algumas estações e meses se destacaram no período de treinamento/ calibração. Destaca-se positivamente os resultados encontrados nas estações fluviométricas de Altamira e Pousada Matrinxa, onde os valores do coeficiente de correlação foram superiores a $0,50 \mathrm{em}$ todos os meses do ano, indi-

Tabela 3 - Coeficientes de correlação $(C C)$ mensais entre as vazões simuladas e observadas nas estações fluviométricas na BHX, no período de treinamento/calibração.

\begin{tabular}{lcccccccccccc}
\hline \multicolumn{1}{c}{ Estação } & Jan & Fev & Mar & Abr & Mai & Jun & Jul & Ago & Set & Out & Nov & Dez \\
\hline Altamira $^{*}$ & 0,84 & 0,70 & 0,75 & 0,75 & 0,91 & 0,82 & 0,60 & 0,56 & 0,66 & 0,53 & 0,75 & 0,70 \\
Pedra do Ó & 0,89 & 0,71 & 0,74 & 0,67 & 0,83 & 0,81 & 0,66 & 0,48 & 0,81 & 0,36 & 0,75 & 0,73 \\
Cajueiro & 0,60 & 0,68 & 0,87 & 0,68 & 0,86 & 0,67 & 0,65 & 0,46 & 0,73 & 0,23 & 0,76 & 0,76 \\
Boa Esperança & 0,58 & 0,75 & 0,49 & 0,72 & 0,78 & 0,85 & 0,79 & 0,51 & 0,67 & 0,41 & 0,55 & 0,77 \\
Jusante do Rio Preto & 0,61 & 0,58 & 0,81 & 0,55 & 0,57 & 0,69 & 0,57 & 0,49 & 0,78 & 0,71 & 0,57 & 0,75 \\
Consul & 0,40 & 0,63 & 0,51 & 0,49 & 0,40 & 0,54 & 0,61 & 0,60 & 0,32 & 0,70 & 0,41 & 0,63 \\
Fazenda Itaguaçu & 0,68 & 0,57 & 0,78 & 0,60 & 0,73 & 0,41 & 0,57 & 0,59 & 0,49 & 0,77 & 0,64 & 0,49 \\
Pousada Matrinxa & 0,73 & 0,79 & 0,86 & 0,61 & 0,78 & 0,78 & 0,56 & 0,84 & 0,69 & 0,84 & 0,90 & 0,67 \\
\hline
\end{tabular}

*Estação de referência no exutório da BHX, utilizada na validação do modelo chuva-vazão. 
cando uma correlação de moderada a forte na simulação da vazão média mensal nestas localidades.

Destaca-se ainda na avaliação mensal mostrada na tabela acima, os resultados encontrados nos meses de fevereiro e julho, onde em todas as estações fluviométricas da BHX foram observadas correlações moderadas a fortes entre as vazões simuladas e observadas, com valores de $C C \geq 0,50$. Valores máximos no coeficiente de correlação, $C C \geq 0,90$ foram observados nas estações de Altamira e Pousada Matrinxa nos meses maio e novembro respectivamente, indicando uma correlação muito forte nestes meses entre as vazões observadas e simuladas.

Aleatoriamente, em alguns poucos meses os resultados apresentaram uma correlação fraca nas simulações das vazões na BHX, principalmente na estação de Cajueiro no mês de outubro, onde o coeficiente de correlação apresentou valor de $C C=0,23$. Em geral, o trimestre agosto/ setembro/outubro (período de estiagem), mostrou o pior desempenho nas simulações das vazões mensais durante o período de treinamento/calibração (exceto na estação fluviométrica em Pousada Matrinxa). Ainda é possível identificar que a estação fluviométrica de Consul apresentou valores no coeficiente de correlação $C C \leq 0,70 \mathrm{em}$ todos os meses do ano, indicando uma correlação moderada entre as vazões simuladas e observadas, com valores de $C C$ abaixo 0,5 em alguns meses.

Para apoiar a análise dos resultados, informações descritivas das vazões mensais para o período de 1981 a 2010 nas estações fluviométricas da BHX são apresentadas na Tabela 4. A estação de Altamira concentra as contribuições de vazões de todas os demais rios na BHX, onde são observados os maiores volumes de vazões mensais com média anual de $8114 \mathrm{~m}^{3} / \mathrm{s}$. Nas estações Consul e Fazenda Itaguaçu são observados os menores volumes de vazões com média anual de 116 e $81 \mathrm{~m}^{3} / \mathrm{s}$ respectivamente.

Tabela 4 - Informações descritivas das series de vazões mensais para o período de 1981 a 2010 nas estações fluviométricas da BHX.

\begin{tabular}{|c|c|c|c|c|}
\hline Estação & Rio & $\begin{array}{l}\text { Mínima } \\
\left(\mathrm{m}^{3} / \mathrm{s}\right)\end{array}$ & $\begin{array}{l}\text { Média } \\
\left(\mathrm{m}^{3} / \mathrm{s}\right)\end{array}$ & $\begin{array}{l}\text { Máxima } \\
\left(\mathrm{m}^{3} / \mathrm{s}\right)\end{array}$ \\
\hline Altamira $^{*}$ & Xingu & 770 & 8114 & 29088 \\
\hline Pedra do Ó & Iriri & 65 & 2421 & 9883 \\
\hline Cajueiro & Curuá & 36 & 805 & 3354 \\
\hline Boa Esperança & Fresco & 40 & 895 & 3851 \\
\hline $\begin{array}{l}\text { Jusante do Rio } \\
\text { Preto }\end{array}$ & $\begin{array}{l}\text { Comandante } \\
\text { Fontoura }\end{array}$ & 16 & 210 & 707 \\
\hline Consul & Van den Steinen & 51 & 116 & 233 \\
\hline $\begin{array}{l}\text { Fazenda Ita- } \\
\text { guaçu }\end{array}$ & Rio Ronuro & 37 & 81 & 223 \\
\hline $\begin{array}{l}\text { Pousada } \\
\text { Matrinxa }\end{array}$ & Culuene & 35 & 264 & 1514 \\
\hline
\end{tabular}

Na Tabela 5 são apresentadas as métricas estatísticas selecionadas para avaliar o desempenho da simulação das vazões mensais no período de verificação entre 2011 e 2016 nas estações fluviométricas da BHX. O modelo conseguiu representar bem as vazões mensais observadas nas estações selecionadas neste período. O coeficiente de correlação ficou acima de 0,80 e o Coeficiente de Nash-Sutcliffe acima de 0,59 em todas as estações avaliadas, indicando uma correlação de moderada a forte entre as vazões simuladas e observadas na BHX.

Uma métrica importante avaliada o VIES, apresentou valores negativos nas simulações no período de verificação, apontando para uma subestimativa discreta do modelo, exceto na estação fluviométrica Pousada Matrinxa, onde o VIES foi positivo igual a $49 \mathrm{~m}^{3} / \mathrm{s}$, indicando superestimativa discreta na simulação das vazões mensais nesta estação (Tabela 5). A REQM apresentou valores baixos em relação ao volume de vazão mensal observado em cada estação da BHX. Destaca-se na Tabela 5 os menores valores da $R E Q M$, de 12 e $26 \mathrm{~m}^{3} / \mathrm{s}$, nas estações Fazenda Itaguaçu e Consul respectivamente.

Ainda na etapa de validação da modelagem chuvavazão aplicada são apresentadas as vazões mensais simuladas e observadas referente ao período entre 2011 a 2014 na estação fluviométrica de Altamira no rio principal Xingu, que representa a estação de referência no exutório da BHX (montante do reservatório de Belo Monte). Assim como, o gráfico de dispersão entre as vazões mensais simuladas e observadas e os Coeficientes de Correlação $(C C)$, Coeficiente de Nash-Sutcliffe $(C N)$, VIES, e a Raiz do Erro Quadrático Médio (REQM), para estação fluviométrica de Altamira na BHX.

Tabela 5 - Métricas Estatísticas entre as vazões mensais simuladas e observadas no período de validação nas estações fluviométricas da BHX.

\begin{tabular}{lcccccc}
\hline Estação & Rio & Período & $C C$ & $C N$ & VIES & REQM \\
\hline Altamira $^{*}$ & Xingu & $2011-$ & 0,95 & 0,90 & -343 & 2352 \\
& & 2014 & & & & \\
Pedra do Ó & Iriri & $2011-$ & 0,93 & 0,84 & -317 & 1001 \\
Cajueiro & & 2014 & & & & \\
& Curuá & $2011-$ & 0,84 & 0,68 & -93 & 301 \\
Boa Esper- & & 2016 & & & & \\
ança & Fresco & $2011-$ & 0,85 & 0,70 & -176 & 484 \\
Jusante do Rio & Comandante & $2011-$ & 0,96 & 0,83 & -46 & 76 \\
Preto & Fontoura & 2014 & & & & \\
Consul & Van den Steinen & $2011-$ & 0,95 & 0,70 & -20 & 26 \\
& & 2014 & & & & \\
Fazenda Ita- & Rio Ronuro & $2011-$ & 0,80 & 0,59 & -1 & 12 \\
guaçu & & 2016 & & & & \\
Pousada & Culuene & $2011-$ & 0,87 & 0,74 & 49 & 111 \\
Matrinxa & & 2016 & & & & \\
\hline
\end{tabular}

*Estação de referência no exutório da BHX, utilizada na validação do modelo chuva-vazão. 
Para melhor entender o comportamento entre a precipitação e a vazão mensal em Altamira foi elaborada a climatologia destas variáveis com dados observados das estações meteorológica do INMET e fluviométrica da ANA para o período de 1981 a 2010 (Fig. 2). A Fig. 2 mostra um regime de chuva bem definido em Altamira, um período menos chuvoso entre os meses de junho a novembro, onde no trimestre agosto/setembro/outubro são registrados os menores volumes de chuva com valores climatológicos mensais abaixo de $50 \mathrm{~mm}$, e um período chuvoso entre os meses de dezembro a maio, onde no trimestre fevereiro, março e abril são registrados os maiores volumes de chuva, com valores climatológicos mensais acima de $300 \mathrm{~mm}$.

A climatologia da vazão mensal em Altamira segue comportamento similar ao da precipitação, com um período de vazante entre os meses de julho a dezembro e um período de cheia entre os meses de janeiro a junho. Conforme demostrado na Fig. 2, o mês de abril apresenta o volume máximo de vazão observado, aproximadamente $20000 \mathrm{~m} / \mathrm{s}$ e o mês de setembro apresenta o volume mínimo de vazão observado, aproximadamente $1200 \mathrm{~m}^{3} / \mathrm{s}$. De acordo com a climatologia o pico de vazão observado em abril, em geral ocorre posteriormente ao mês com maior volume de chuva em março. Fica evidente em Altamira a relação e dependência da vazão do mês (t) com a chuva do mês anterior $(t-1)$, principalmente no período chuvoso e de transição dezembro-janeiro e maio-junho.

O grau de relação entre precipitação e vazão nos meses de pico (cheia e estiagem) na estação fluviométrica de referência em Altamira ficou demostrado na Fig. 2. Devido ao ciclo sazonal da chuva na Amazônia os rios apresentam os seus níveis mais altos poucos meses após as chuvas mais intensas. Consequentemente, na Amazônia períodos de seca meteorológica com poucas chuvas na estação chuvosa, são seguidos por períodos de seca hidrológica, com níveis baixos dos rios na estação de cheia (Marengo e Espinosa, 2015).

A evolução mensal da cota fluviométrica do Rio Xingu em Altamira associada aos eventos de El Niño e La Niña no período de 1979 a 2012 foi avaliada por Franco et al., 2009. Para anos de atuação de El Niño, os resultados evidenciaram padrões de convecção tropical anomalamente enfraquecidos nas regiões de atuação da ZCIT e da ZCAS, resultando consequentemente na ocorrência de anomalias negativas de precipitação em grande parte da Amazônia, explicando, assim, o regime hidrológico predominantemente abaixo da média no rio Xingu em Altamira-PA. Já para anos de La Niña, essas anomalias no padrão da convecção tropical nestas regiões se inverte, e o regime hidrológico observado predomina acima da média no Rio Xingu em Altamira.

As vazões mensais simuladas e observadas na estação fluviométrica em Altamira no período de validação são mostradas na Fig. 3. De uma maneira geral, o modelo consegui simular muito bem as vazões médias mensais, principalmente nos meses de estiagem (junho a novembro). Nos meses de fevereiro, março e abril, onde ocorre os eventos de cheia, o modelo conseguiu capturar o mês com a vazão máxima observada em praticamente todo o período, exceto no ano de 2014. Na maioria dos meses no período de cheia, houve uma subestimativa discreta do modelo, com exceção no mês de abril de 2013, onde o modelo superestimou a vazão mensal observada em função da precipitação acumulada. Modelos hidrológicos devem ser aplicados e avaliados com cautela no período chuvoso, onde ocorrem os eventos extremos de precipitação e consequentemente vazões de pico (Lucas et al., 2009).

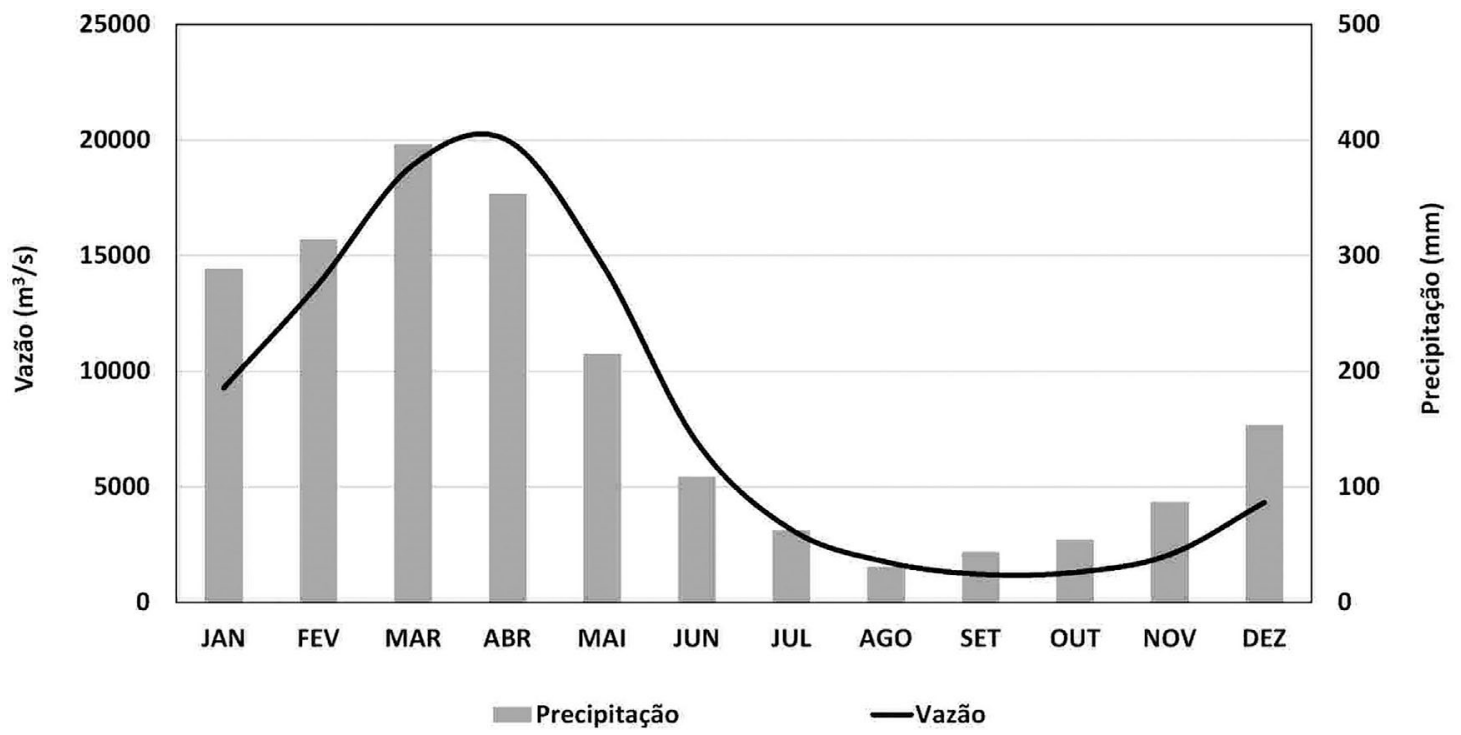

Figura 2 - Climatologia mensal da precipitação e vazão em Altamira no período de 1981 a 2010. 


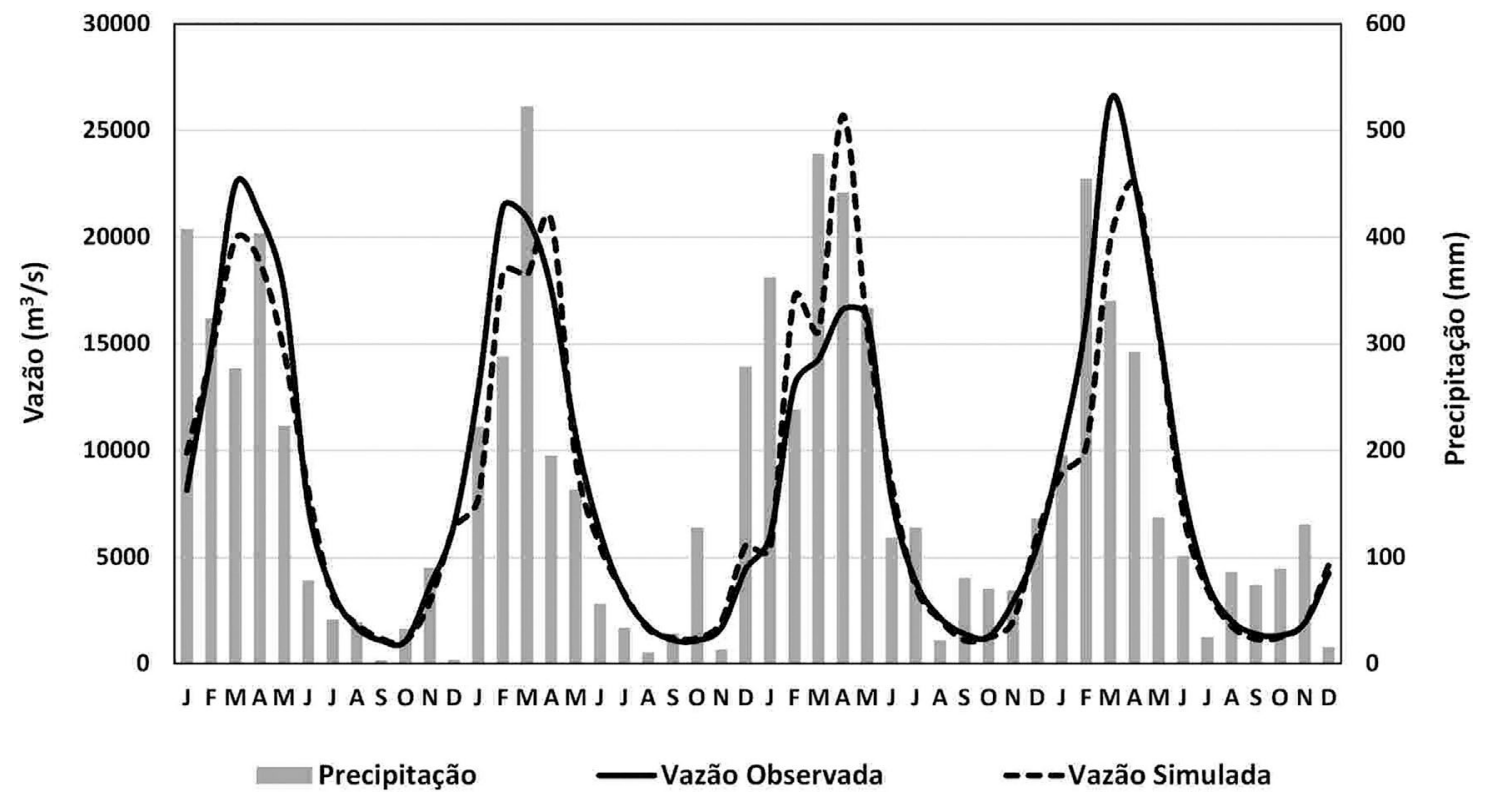

Figura 3 - Precipitação mensal observada e vazões mensais simuladas e observadas na estação de Altamira, período de 2011 a 2014 (validação).

De acordo com o gráfico de dispersão entre as vazões médias mensais simuladas e observadas na estação fluviométrica de Altamira no período de validação (Fig. 4), os resultados foram considerados satisfatórios, com os valores dos indicadores de desempenho selecionados de coeficiente de correlação $(C C)$ igual a 0,96 e o coeficiente de Nash-Suticliffe $(C N)$ igual a 0,90 . De acordo com a literatura, os resultados encontrados nestes indicadores apontam para uma correlação muito forte entre a vazão simulada e observada em Altamira. O erro (VIES) apresentou um valor negativo discreto de $-343 \mathrm{~m}^{3} / \mathrm{s}$, demostrando subestimativa nas simulações das vazões mensais. A REQM apresentou o valor de $2343 \mathrm{~m}^{3} / \mathrm{s}$, considerado baixo em relação ao grande volume de vazão mensal observado na estação fluviométrica de Altamira.

Lucas et al. (2009), simularam a vazão mensal na região hidrográfica do Xingu no Pará, aplicando um modelo determinístico de dois parâmetros e o modelo estocástico ARIMA. O modelo determinístico com uma estrutura simples apresentou bons resultados, porém mostrou-se sensível a eventos extremos de precipitação. O modelo estocástico ARIMA, conseguiu capturar a dinâmica das séries temporais, apresentando melhores resultados na simulação comparado ao modelo determinístico, onde os coeficientes de Nash-Sutcliffe apresentaram valores de 0,73 e 0,90 respectivamente na estação de Altamira. Os resultados do modelo empírico chuva-vazão aplicado neste trabalho, corroboram com estudo de Lucas et al. (2009), no desempenho satisfatórios desta categoria de modelo na simulação da vazão mensal na BHX.

Malfatti et al. (2008); desenvolveram modelos empíricos de Regressão Linear Múltipla para previsão de vazões mensais defasadas no tempo na Usina Hidrelétrica de Itaipu - Bacia do Rio Paraná. De um modo geral, os modelos validados indicaram melhor desempenho nas defasagens mais curtas quando considerado os preditores de vazão e precipitação. Para preditores climáticos como Índices de El Niño Oscilação Sul e anomalias de temperatura da superfície do mar no Atlântico Tropical Sul, os modelos mostraram melhores resultados nas defasagens mais longas. Para os autores, os modelos empíricos são ferramentas uteis, pois podem ser facilmente ajustados a diferentes localidades, períodos e conjunto de dados. Estes resultados reforça a aplicação satisfatória de modelos

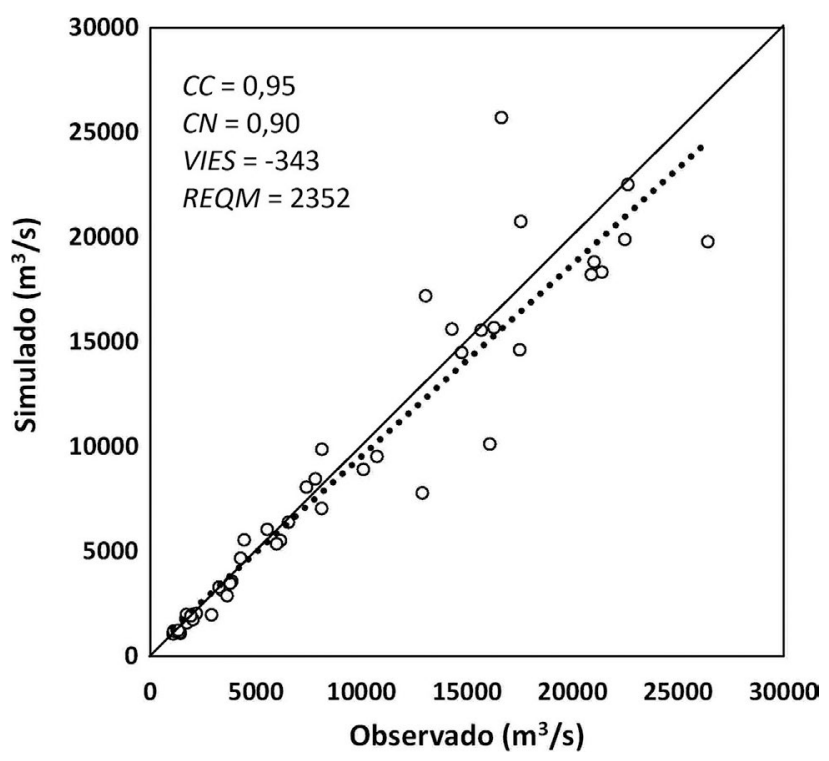

Figura 4 - Dispersão entre as vazões mensais simuladas e observadas na estação de Altamira no período de 2011 a 2014 (validação). 
empíricos na simulação de vazões mensais em grandes bacias com aproveitamento energético, como demostrado neste trabalho na BHX.

Villar et al. (2009), mostraram que mudanças extremas nas descargas dos rios da bacia Amazônica estão associadas com a variabilidade regional da precipitação e a circulação atmosférica, bem como, aos indicadores climáticos tropicais de grande escala. Com base nestes resultados, previsões climáticas sazonais de precipitação confiáveis que consideram as influencias remotas do clima (como as previsões disponibilizadas pelos Serviços Meteorológico Nacionais e Instituo de Pesquisas), podem ajudar a melhorar os resultados das simulações das vazões mensais dos modelos hidrológicos do tipo chuva-vazão, como neste modelo empírico aplicado neste trabalho.

\section{Conclusões}

Ficou demostrado o grau de relação entre precipitação e vazão mensal nas estações fluviométricas da BHX, identificando-se o número apropriado de defasagens de precipitação como entrada no modelo chuva-vazão para cada estação. A partir das simulações realizadas, verificou-se que para estações fluviométricas localizadas em grandes áreas de drenagem a vazão mensal em um determinado mês $(t)$, está mais relacionada com as chuvas do mês anterior $(t-1)$. Já estações fluviométricas localizadas em áreas de drenagem menores mostraram vazões mensais mais correlacionadas com as precipitações do mesmo mês $t(0)$.

A RCP aplicada à simulação das vazões mensais apresentou bons resultados em todas as estações selecionadas. Na estação fluviométrica de Altamira, referência da $\mathrm{BHX}$, os resultados foram promissores e satisfatórios, com o modelo conseguindo capturar a dinâmica da série temporal e mostrando excelente desempenho nas simulações das vazões nos meses de estiagem (maio a outubro), porém, apresentou uma subestimativa discreta no período chuvoso (novembro a abril).

Com base nos resultados, a modelagem empírica chuva-vazão aplicada utilizando RCP atende uma demanda de previsões menos complexas, mais rápidas e confiáveis principalmente em grandes bacias como na Amazônia, com problemas de disponibilidade de dados de entrada dos modelos conceituais, mostrando-se uma ferramenta útil e operacional nas simulações de vazões mensais na BHX.

Previsões de vazões mensais utilizando RCP podem ser realizadas com dados de precipitação mensal disponibilizados de previsões climáticas sazonais e/ou cenários climáticos, sendo capazes de auxiliar e apoiar a tomada de decisões em setores-chave, como planejamento da geração de energia elétrica na usina hidroelétrica Belo Monte e no gerenciamento dos usos múltiplos dos recursos hídricos na BHX.

\section{Agradecimentos}

Os autores agradecem ao Instituto Nacional de Meteorologia - INMET e a Agência Nacional de Águas - ANA, pelo fornecimento de dados e apoio no desenvolvimento do trabalho. Os revisores anônimos são reconhecidos por seus valiosos comentários e sugestões.

\section{Referências}

AleXANDre, A.M.B. Previsão de Vazões Mensais Para o Sistema Interligado Nacional Utilizando Informações Climáticas. Tese de Doutorado em Engenharia Civil, Departamento de Engenharia Hidráulica e Ambiental, Universidade Federal do Ceará, 2012.

ALMEIDA, L.; SERRA, J.C.V. Hydrological models, types and applications used more. Revista da FAE, v. 20, n. 1, p. 129-137, 2017.

ANA - AGENCIA NACIONAL DE AGUAS. HIDROWEB v3.1.1 - Séries Históricas de Estações. Disponível em http://www.snirh.gov.br/hidroweb/serieshistoricas, acesso em 5 de ago. de 2019, 2018.

BISHOP, C.M. Pattern Recognition and Machine Learning. New York: Springer, 758 p., 2006.

BOZZINI, P.L.; MELLO JUNIOR, A.V. Previsões de precipitação de modelos atmosféricos como subsídio à operação de sistemas de reservatório. Revista Brasileira de Meteorologia, v. 35, n. 1, p. 99-109, 2020.

BRAVO, J.M.; ALLASIA, D.; PAZ, A.R.; COLLISCHONN, W.; TUCCI, C.E.M. Coupled hydrologic-hydraulic modeling of the upper Paraguay River Basin. Journal of Hydrologic Engineering, v. 17, n. 5, p. 635-646, 2012.

CÂMARA, R.K.C.; ROCHA, E.J.P.; PROTÁZIO, J.M.B.; QUEIROZ, J.C.; RIBEIRO, W.M.N. et al. Hydrological stochastic modeling applied to the Tocantins River to the city of Marabá (PA). Revista Brasileira de Meteorologia, v. 31, n. 1, p. 11-23, 2016.

COE, M.T.; COSTA, M.H.; SOARES-FILHO, B.S. The influence of historical and potential future deforestation on the stream flow of the Amazon River - Land surface processes and atmospheric feedbacks. Journal of Hydrology, v. 369, n. 1-2, p. 165-174, 2009.

CPRM. Bacia do Xingu - Características. Disponível em: https://www.cprm.gov.br/sace/xingu_caracteristicas.php, acesso em 5 ago. 2019, 2019.

DA SILVA, P.E.; SILVA, C.M.S.; SPYRIDES, M.H.C.; ANDRADE, L.M.B. Precipitation and air temperature extremes in the Amazon and northeast Brazil. International Journal of Climatology, v. 39, n. 2, p. 1-17, 2018.

DA SILVA, P.E.; SANTOS E SILVA, C.M.; SPYRIDES, M.H.C.; ANDRADE, L.M.B. Analysis of climate extreme indices in the Northeast Brazil and the Brazilian Amazon in the period from 1980 to 2013. Anuário do Instituto de Geociências - UFRJ, v. 42, n. 2, p. 137-148, 2019.

DE MENEZES, J.B.; BANDEIRA, O.M.; LEITE, D.T. A construção do complexo hidrelétrico de Belo Monte: quarta maior do mundo em capacidade instalada. Revista Brasileira de Engenharia de Barragens - Edição Especial Belo Monte, v. 4, p. 5-21, 2017. 
DEVI, G.K.; GANASRI, B.P.; DWARAKISH, G.S. A Review on Hydrological Models. Aquatic Procedia, v. 4, n.1, p. 1001-1007, 2015.

DIAS, V.S.; LUZ, M.P.; MEDERO, G.M.; NASCIMENTO, D.T.F. An overview of hydropower reservoirs in Brazil: current situation, future perspectives and impacts of climate change. Water, v. 10, n. 5, p. 1-18, 2018.

DUAN, L.; LIU, T.; WANG, X.; LUO, Y.; WU, L. Development of a regional model estimating annual runoff in the Hailar river basin of China. Journal Water Resource and Protection, v. 2, n. 11, p. 934-943, 2010.

ESQUIVEL, A.; LLANOS-HERRERA, L.; AGUDELO, D.; PRAGER, S.D.; KATIA FERNANDES; ROJAS, A.; VALENCIA, J.J.; RAMIREZ-VILLEGAS, J. Predictability of seasonal precipitation across major crop growing areas in Colombia. Climate Services, v. 12, n. 4, p. 36-47, 2018.

FILHO, F.A.S.; LALL, U. Modelo de previsão de vazões sazonais e interanuais. Revista Brasileira de Recursos Hídricos, v. 22, n. 2, p. 61-74, 2004.

FRANCO, V.S.; SOUZA, E.B.; LIMA, A.M.M.; SOUZA, A.L.; PINHEIRO, A.N.; DIAS, T.S.; AZEVEDO, F.M. Climatologia e previsão hidrológica de cheia sazonal do rio Xingu, Altamira-PA. Revista Brasileira de Climatologia, v. 22, n. 1, p. 1-19, 2018.

HOTELLING, H. The relations of the newer multivariate statistical methods to factor analysis. British Journal of Mathematical Statistical Psychology, v. 10, n. 2, p. 69-79, 1957.

HU, T.; WU, F.; ZHANG, X. Rainfall-runoff modeling using principal component analysis and neural network. Nordic Hydrology, v. 38, n. 3, p. 235-248, 2007.

IRI - INTERNATIONAL RESEARCH INSTITUTE FOR CLIMATE AND SOCIETY. The Climate Predictability Tool. Disponível em https://iri.columbia.edu/our-expertise/climate/tools/cpt/, 2019, acesso em 5 ago. de 2019.

IZENMAN, A.J. Modern Multivariate Statistical Techniques. New York: Springer, 758 p., 2008.

JOLLIFFE, I.T. A note on the use of principal components in regression. Applied Statistics, v. 31, n. 3, p. 300-303, 1982.

KENDALL, M.G. Rank Correlation Methods. London: Charles Griffin, 120 p., 1975.

KIPKOGEI, O.; MWANTHI, A.M.; MWESIGWA, J.B., ATHERU, Z.K.K.; WANZALA, M.A.; ARTAN. G. Improved seasonal prediction of rainfall over East Africa for application in agriculture: statistical downscaling of CFSv2 and GFDL-FLOR. Journal of Applied Meteorology and Climatology, v. 56, n 12, p. 3229-3243, 2017.

LANDMAN, W.A.; BARNSTON, A.G.; VOGEL, C.; SAVY, J. Use of El Niño-Southern Oscillation related seasonal precipitation predictability in developing regions for potential societal benefit. International Journal of Climatology, v. 39, n. 14, p. 5327-5337, 2019.

LUCAS, E.W.M.; SOUSA, F.A.S.; SILVA, F.D.S.; LUCIO, P.S. Modelagem hidrológica determinística e estocástica aplicada à Região Hidrográfica do Xingu - Pará. Revista Brasileira de Meteorologia, v. 24 n. 3, p. 308-322, 2009.

LUCIO, P.S., et al. Um modelo estocástico combinado de previsão sazonal para a precipitação no Brasil. Revista Brasileira de Meteorologia, v. 25, n. 1, p. 70-87, 2010.
MALFATTI, M.G.L.; LINGIARDI, A.M.; CARDOSO, A.O. Modelo empírico para previsão de vazão de rios - Caso Itaipu. In: Congresso Brasileiro de Meteorologia, RecifePE, SBMET, 2014.

MALFATTI, M.G.L.; CARDOSO, A.O; HAMBURGER, D.S. Modelo empírico linear para Previsão de vazão de Rios na Usina Hidrelétrica de Itaipu - Bacia do Rio Paraná. Revista Brasileira de Meteorologia, v. 33, n. 2, p. 257-268, 2018.

MARENGO, J.A.; ESPINOZA, J.C. Review Extreme seasonal droughts and floods in Amazonia: Causes, trends and impacts. International Journal of Climatology, v. 36, n. 3, p. 1033-1050, 2015.

MASON, S.J., TIPPETT, M.K. Climate Predictability Tool version 15.5.10, 2017. https://academiccommons.columbia.edu/doi/10.7916/D8G44WJ6.

MORIASI, D.N.; ARNOLD, J.G.; VAN LIEW, M.W.; BINGNER, R.L.; HARMEL, R.D.; VEITH. T.L. Model evaluation guidelines for systematic quantification of accuracy in watershed simulations. Transactions of the ASABE, v. 50, n.3, p. 885-900, 2007.

NASH, J.E.; SUTCLIFF, J. River flow forecasting through conceptual models. Journal of Hydrology, v. 10, n. 3, p. 282290, 1970.

NORTE ENERGIA. UHE Belo Monte, A Maior Usina Hidrelétrica $\mathbf{1 0 0 \%}$ Brasileira. Brasília: Norte Energia. Disponível em https://www.norteenergiasa.com.br/pt-br/uhebelo-monte/a-usina, acesso em ago. 2019, 2018.

OTTO, M. Chemometrics. Weinheim: Wiley, 328 p., 1999.

PAREDES-TREJO, F.; BARBOSA, H.; DOS SANTOS, C.A.C. Evaluation of the performance of SM2RAIN-derived rainfall products over Brazil. Remote Sensing, v. 11, n. 1113, p. 1-28, 2019.

REBOITA, M.S.; GAN, M.A.; DA ROCHA, R.P.; AMBRIZZI, T. Regimes de precipitação na América do Sul: Uma revisão bibliográfica. Revista Brasileira de Meteorologia, v. 25, n. 2, p. 185-204, 2010.

REMESAN, R.; SHAMIM, M.A.; HAN, D.; JIMSON, M. Runoff prediction using an integrated hybrid modelling scheme. Journal of Hydrology, v. 372, n. 1-4, p. 48-60, 2009.

REMESAN, R.; BRAY, M.; J. MATHEW. J. Application of PCA and Clustering Methods in input selection of Hybrid Runoff Models. Journal of Environmental Informatics, v. 31, n. 2, p. 137-152, 2018.

SANTOS, E.B.; LUCIO, P.S.; SANTOS E SILVA, C.M. Synoptic patterns of atmospheric circulation associated with intense precipitation events over the Brazilian Amazon. Theoretical and Applied Climatology, v. 128, n. 1-2, p. 343-358, 2015.

SANTOS, C.A.; LIMA, A.M.M.; FRANCO, V.S.; ARAÚJO, I.B.; MENEZES, J.F.G.; GOMES, N.M. O. Distribuição espacial da precipitação na bacia hidrográfica do rio Xingu. Nucleus, v. 13, n. 2, p. 223-230, 2016.

SILVA, J.P.; PEREIRA, D.I.; AGUIAR, A.M.; RODRIGUES, C. Geodiversity assessment of the Xingu drainage basin. Journal of Maps, v. 9, n. 2, p. 254-262, 2013.

STICKLER, C.M.; COE, M.T.; COSTA, M.H.; NEPSTAD, D.C.; MCGRATH, D.G.; DIAS, L.C.P.; RODRIGUES, H.O.; SOARES-FILHO, B.S. Dependence of hydropower 
energy generation on forests in the Amazon Basin at local and regional scales. PNAS, v. 4, n. 110, p. 9601-9609, 2013.

TAYLOR, R. Interpretation of the correlation coefficient: A basic review. Journal of Diagnostic Medical Sonography, v. 6, n. 1, p. 35-39, 1990.

TODINI, E. Hydrological catchment modelling: Past, present and future. Hydrology and Earth System Sciences, v. 11, n. 1, p. 468-482, 2007.

TUCCI, C.E.M. Modelos Hidrológicos. Porto Alegre: UFRGS/ ABRH, 678 p., 2005.

VILLAR, J.C.E.; GUYOT, J.L.; RONCHAIL, J.; COCHONNEAU, G.; FILIZOLA, N.P.; FRAIZY, P.; LABAT, D.; OLIVEIRA, E.; ORDOÑEZ GÁLVEZ, J.J.; VAUCHEL, P. Contrasting regional discharge evolutions in the Amazon Basin (1974-2004). Journal of Hydrology, v. 375, n. 3-4, p. 297-311, 2009.
VILlAS-BÔAS, A. De Olho na Bacia do Xingu. Série Cartô Brasil Socioambiental. São Paulo: ISA, 63 p., 2012.

XAVIER, A.C.; KING, C.W.; SCANLON, B.R. Daily gridded meteorological variables in Brazil (1980-2013). International Journal of Climatology, v. 36, n. 6, p. 2644-2659, 2016.

WAGENER, T.; WHEATER, H.S. Parameter estimation and regionalization for continuous rainfall-runoff models including uncertainty. Journal of Hydrology, v. 320, n. 1-2, p. 132-154, 2006.

WILKS, D.S. Statistical Methods in the Atmospheric Sciences. Philadelphia: Elsevier Academic Press Publications, 626 p., 2006.

License information: This is an open-access article distributed under the terms of the Creative Commons Attribution License (type CC-BY), which permits unrestricted use, distribution and reproduction in any medium, provided the original article is properly cited. 